\title{
Co-simulation of Magnetic Bearing System based on Adams and MATLAB
}

\author{
Sijia Liu ${ }^{1, a}$, Jingbo Shi ${ }^{2, b}$ \\ ${ }^{1}$ School of BEIHANG University, Beijing 100191, China; \\ ${ }^{2}$ School of BEIHANG University, Beijing 100191, China. \\ a15201342733@163.com, '04594@buaa.edu.cn
}

Keywords: magnetic bearings, mathematical model, electromagnetic force model, co-simulation

\begin{abstract}
In this paper, a general mathematical model is shown through the mathematical analysis of the magnetic bearing. The linear expression of relationship between the electromagnetic force, the deviation of the displacement and control current of is provided by the finite element analysis, with Maxwell 3D. Using the resulting mathematical models and the expression of the electromagnetic force to establish the ideal PID control model and adjust its parameters in MATLAB. Lastly, a optimization model is proposed according to the simulation results, using co-simulation interface-based approach in the MATLAB and ADAMS to control a bearing system from a floating state. The conclusion can provide a reference for the design of active magnetic bearing radial control system.
\end{abstract}

\section{Introduction}

Magnetic bearing is a kind of new bearing which use electromagnets controllable electromagnetic force to keep the rotor levitating without any contact. Compared with conventional bearings, magnetic bearings have many advantages, such as no friction, no wear, no pollution, no lubrication and damping adjustable stiffness and so on [1].

With the rapid development of computer technology and all kinds of software, there are different ways for the study and modeling of magnetic bearings. As the traditional mathematical model is too ideal, the multi-body dynamics system has become the most active areas of engineering modeling theory because of its consider of mechanical properties. With development of virtual prototyping technology, the simulation and testing based on virtual prototype has become an important means of research. However, there are always advantages and disadvantages in a single software. Co-simulation makes it possible for two or more emulation software to connect via a bidirectional data interface, with real-time data exchange, so that the virtual model is closer to the actual establishment of the model, using the project has more far-reaching significance [2].

In this paper, the design of the magnetic bearing control system is based on the co-simulation between Adams and MATLAB, with the electromagnetic results in Maxwell 3D. The research process of this paper can be used in other modeling and simulation on the bearing system.

\section{Principle of Magnetic Bearings}

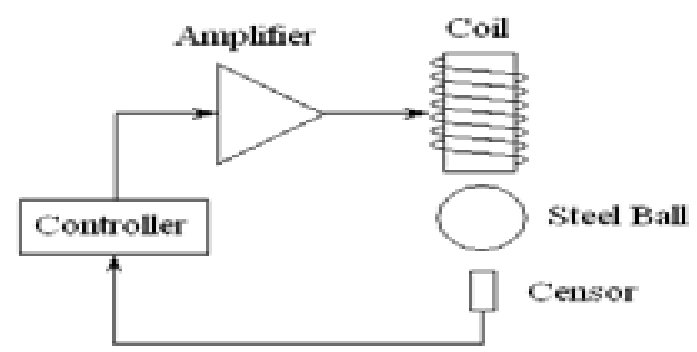

Fig.1 Principle of 1-DOF magnetic bearing 
A complete magnetic bearing rotor system consists of control section and mechanical parts. The control section includes a sensor, a power amplifier, a controller, with mechanical part comprising a rotor and an electromagnet, etc. Fig. 1 shows the block diagram of each degree of freedom in a general magnetic bearing [3]. The position error of the bearing positions is measured by the sensor. The error is processed by a controller, which usually is a PID controller. The output of the controller controls the current of the coil via operational amplifier. Finally the current in the coil produce a electromagnetic force which effect on the bearing, making the bearing closer to the standard position. This process is repeated several times until the magnetic bearing is moved to the standard position.

Generally speaking, a magnetic bearing has a total of five degrees of freedom in the space, four in the radial and one in the axial. For the bearings on low-speed rotation, the coupling between the displacement and currents of various degrees can be ignored. It can be independently controlled for each degree of freedom. However, when the rotation has a high speed, the strong coupling between the various degrees of freedom will make the independent control not practical. In this situation, other control methods should be considered, such as intelligent control, Robust Control and so on.

\section{System Modeling}

\section{Mathematical Model.}

In order to obtain the mathematical model of the magnetic bearing, the relationship between the electromagnetic force and the feedback of the parameters should be deduced first. Using magnetic circuit analysis method, with the magnetic flux leakage ignored in the magnetic circuit of the electromagnets and the rotor and the air gap between them, the relation of the bias current and the magnetic flux in the circuit within the core range is shown.

$$
\begin{aligned}
& \phi=N I \\
& \text { the reluctance of the air gap and cores are given by } \\
& R_{o}=\frac{2 C_{0}}{\mu_{0} A_{a}} \\
& R_{f}=\frac{I_{f}}{\mu_{f} A_{f}}
\end{aligned}
$$

flux is obtained by ampere loop theorem, with magnetization of the core omitted

$$
B=\mu_{0} \frac{N I}{2 C_{0}+\frac{I_{f}}{\mu_{f}}}=\mu_{0} \frac{N I}{2 C_{0}}
$$

Then calculate the electromagnetic force, the magnetic force is generated at the boundary of different permeability of the medium, the electromagnetic force should be given based on field energy theory. Consider the energy $W_{a}$ stored in the air gap in the volume.

$$
W_{a}=\frac{1}{2} B_{a} H_{a} V_{a}
$$

By the principle of virtual displacement, air gap $s$ increased $d s, W_{a}$ in the field of energy changed $d W_{a}$, it is possible to obtain an electromagnetic force.

$$
f=\frac{\partial W_{a}}{\partial s}
$$

In which the magnetic flux density $B$ is a function of the coil current $i$ and the air gap $s$, so the $\mathrm{f}$ can be given by

$$
f=\frac{1}{4} \mu_{0} N^{2} A a \frac{I^{2}}{C_{0}^{2}}
$$

For typical magnetic bearing system, one degree of freedom usually has two opposing electromagnets provide the electromagnetic force, the relationship between force and current can be 
converted into a linear relationship. After the rotor is disturbed, the electromagnetic force provided by two opposite directions is given by

$$
\begin{aligned}
& f_{+}=k \frac{\left(i_{0}+i_{x}\right)^{2}}{\left(s_{0}-x\right)^{2}} \\
& f_{-}=k \frac{\left(i_{0}-i_{x}\right)^{2}}{\left(s_{0}+x\right)^{2}}
\end{aligned}
$$

the composition of forces is given by

$$
f_{x}=f_{+}-f_{-}=k\left(\frac{\left(i_{0}+i_{x}\right)^{2}}{\left(s_{0}-x\right)^{2}}-\frac{\left(i_{0}-i_{x}\right)^{2}}{\left(s_{0}+x\right)^{2}}\right)=4 k\left(\frac{x}{s_{0}}-\frac{i_{x}}{i_{0}}\right)^{2}
$$

The linearized equation is given by

$$
f_{x}=\frac{4 k i_{0}}{S_{0}{ }^{2}} i_{x}+\frac{4 k i_{0}{ }^{2}}{S_{0}{ }^{3}} x=k_{i} i_{x}-k_{x} x
$$

In summary, the force-current relationship can be idealized to a linear relationship of the displacement and the control current, with the disturbance in a lesser extent. The remaining parameters are entirely determined by the magnetic bearing structure itself, such as vacuum magnetic permeability, cross-sectional area of the core, the turns of electromagnet coil, the bias current, the size of the air gap.

Simultaneous equations of the force - current relationship and Newton's laws can be solved as

$$
m \ddot{x}=k_{i} i_{x}-k_{x} x
$$

The transfer function of the displacement and the controlled current can be obtained.

$$
\begin{aligned}
& M s^{2} X(s)=k_{i} I_{x}(s)-k_{x} X(s) \\
& X=\frac{k_{i}}{M s^{2}+k_{x}} I_{x}
\end{aligned}
$$

Obviously, the magnetic bearing is a second-order non-damping system, which is unstable after disturbed in theory [4].

\section{Electromagnetic Force Model.}

Finite element analysis (FEA) using the method of mathematical approximation to simulate for the real physical system (both geometry and load conditions). It means use a limited number of unknown quantities to approximate the infinite unknown real system, with simple but interacting elements. In the finite element software, such as Maxwell 3D, axial suction disk model is created, as shown in Fig. 2(a).

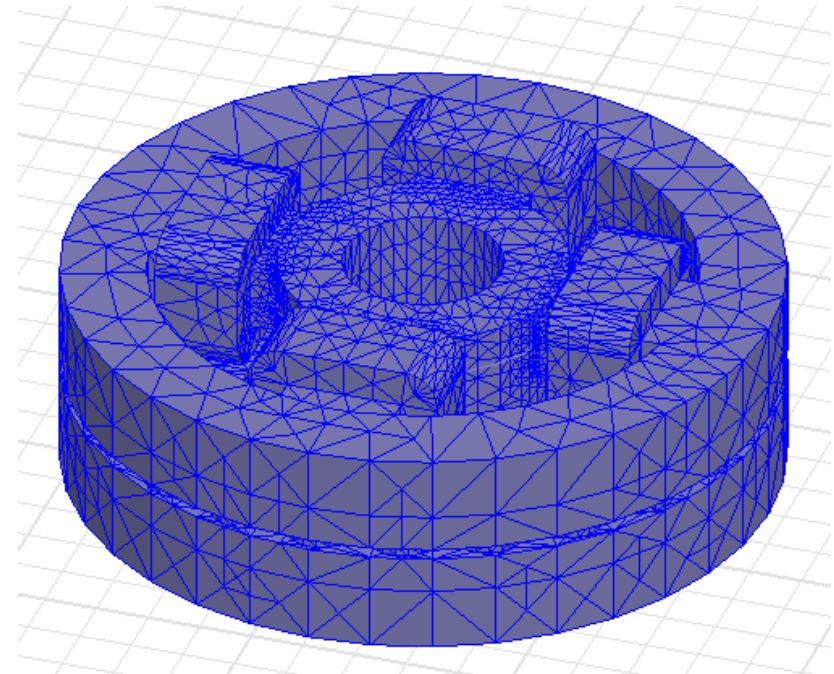

(a) Finite element triangulation of the radial bearings 


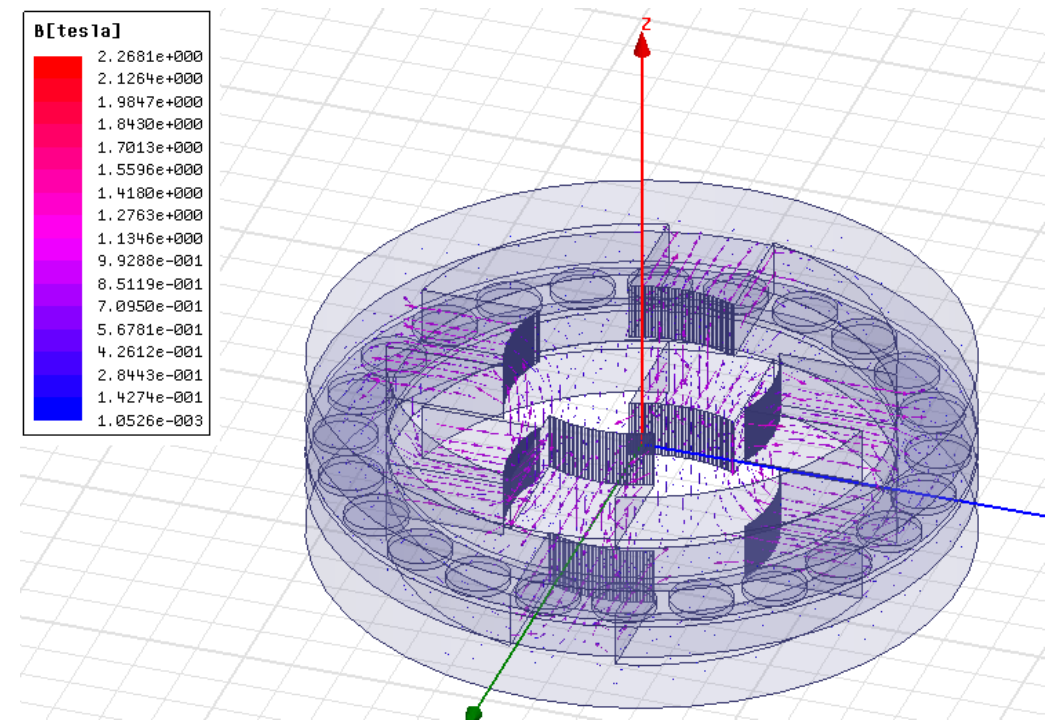

(b) Magnetic flux density with the rotor in the standard position

Fig. 2 the Finite element simulation of the bearing in Maxwell 3D

Fig.2(b) shows the magnetic flux density when the rotor is in the position of equilibrium, when the current of the coil is zero. It can be seen that the path of magnetic flux density is as the same as the analysis of magnetic circuit.

This paper does not consider the effect on the rotor magnetic field caused by the rotation. The model is solved by the static magnetic field solver. The maximum number of steps is set to ten, and error tolerance value is set to one percent, can meet the most basic simulation. After the actual simulation under these conditions, each simulation substantially reaches convergence before the fifth step.

In this paper, the force on the rotor is simulated with the error of the rotor's displacement rotor is set to a single direction at $0.05 \mathrm{~mm}$ to $0.49 \mathrm{~mm}$, and the control current changes between 0 and 270 ampere-turns. The data is shown in Fig.3.

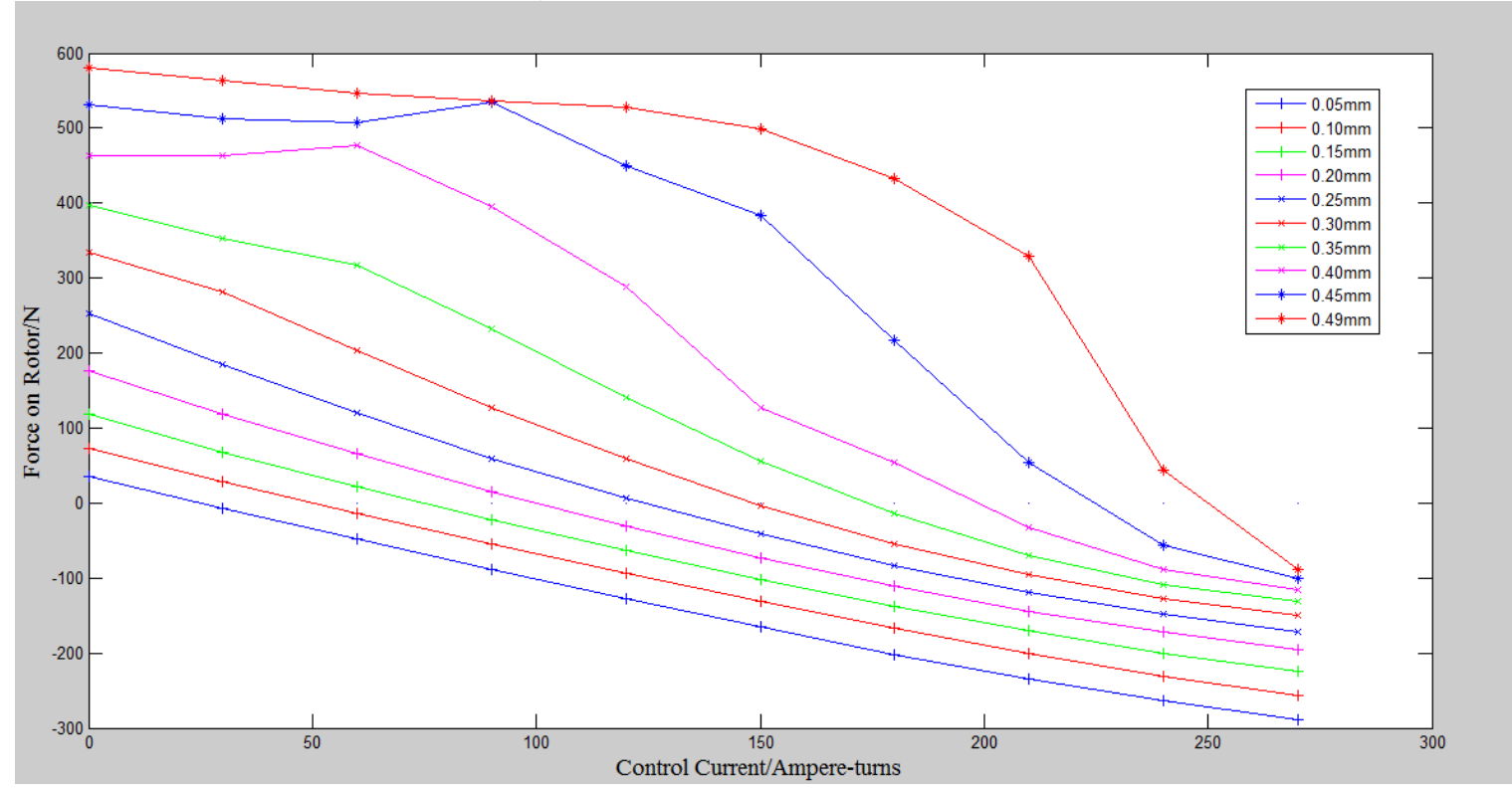

Fig.3 Force on the rotor in different position and current

Since the bearing structure is symmetrical, so the case of the negative direction of displacement is substantially the same, except for the direction.

As can be seen, when the rotor is approach to the standard position, the relationship between electromagnetic force and error of displacement and current is linear. As the position error increases, the relationship becomes complicated. For a simple treatment, only the linear fit of the relationship is more focus on the position near the standard.

Using the CFTOOL in MATLAB, the linearized expression can be given by 
$f_{x}=643.8 x-75 i_{x}$

The linearized expression will be used in the co-simulation.

\section{Simulation with MATLAB and ADAMS}

\section{Simulation with MATLAB.}

A block diagram of a magnetic bearing control system is build in MATLAB, with the parameters of bearing set in the mathematical model expressions obtained before. Adjust the parameters of the control system. The diagram is shown in Fig.4(a),result in Fig.4(b).

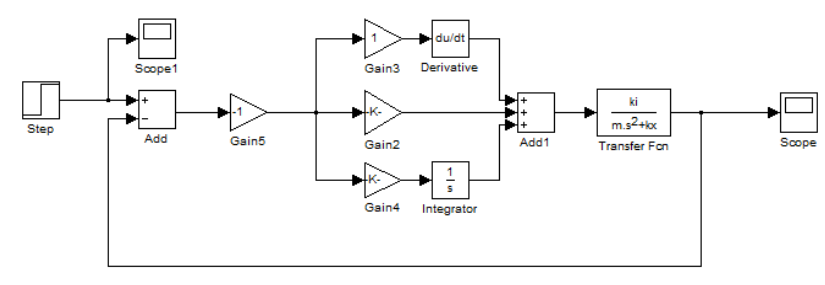

(a) Block diagram of control system

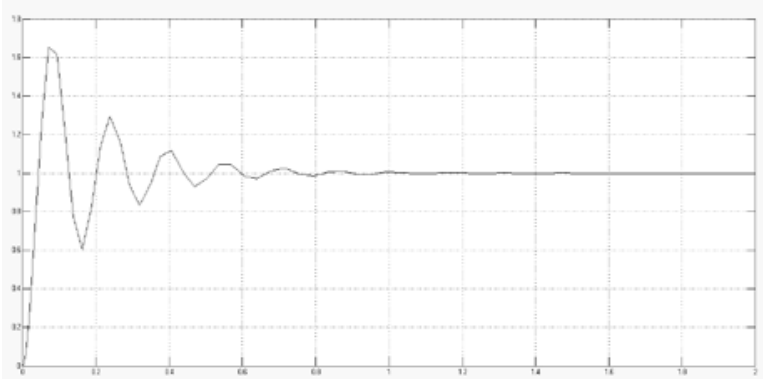

(b) Result of simulation

Fig.4 Simulation of control system in MATLAB

Fig.4 shows a stable control system, in which $K_{p}$ equals to $-200, K_{i}$ equals to $-1, K_{d}$ equals to -0.1 .

\section{Co-simulation with MATLAB and ADAMS.}

The co-simulation between MATLAB and ADAMS via a bidirectional data interface need a mechanical model of the bearing in ADAMS and a control system in MATLAB. The mechanical model is shown in Fig.5, in which the rotor is the blue one, the radial bearings are red, the axial is green.

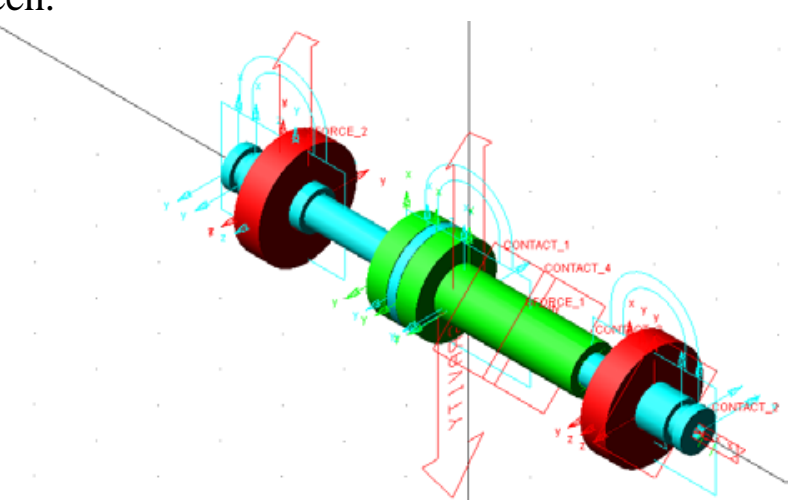

(a) All parts of the model

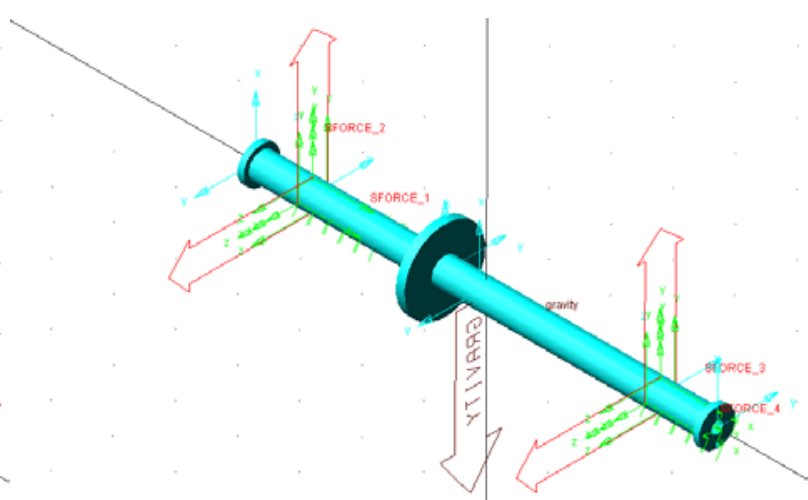

(b) Rotor and reference point

Fig. 5 Mechanical model of the bearing in ADAMS

In the mechanical model, eight reference point were set to simulate the situation that the rotor is not on the standard position [5]. For the mechanical model, the output variables are the error of displacement, which is set as $-0.1 \mathrm{~mm}$ and $-0.2 \mathrm{~mm}$. The input variables are the forces working on the rotor, shown as SFORCE_1 to SFORCE_4 in Fig.5(b).

The control system is build in MATLAB, shown in Fig.6(a). Fig.6(b) shows the Independent control system for each degree of freedom, with the parameters adjusted above. The Gain 4 and 5 is determined by the linear expression of the electromagnetic force, which is shown by formula (15). 


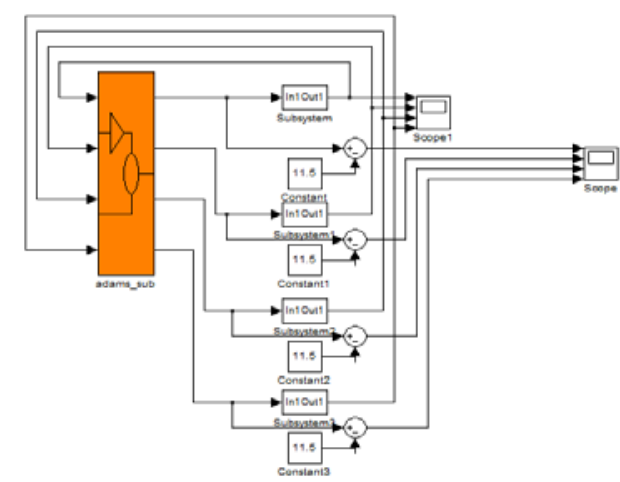

(a) Control system of co-simulation

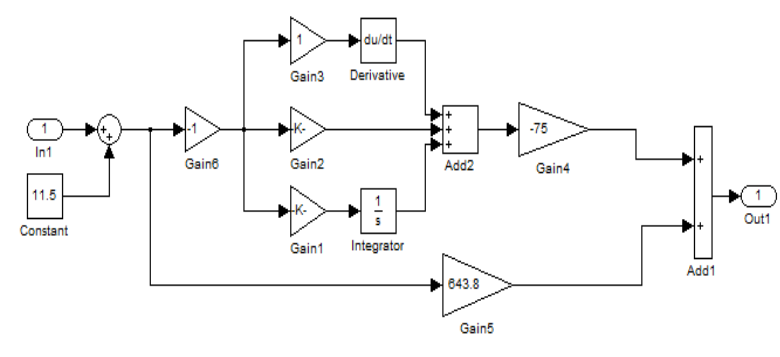

(b) Control system for each degree of freedom

Fig.6 Control system of the bearing in MATLAB

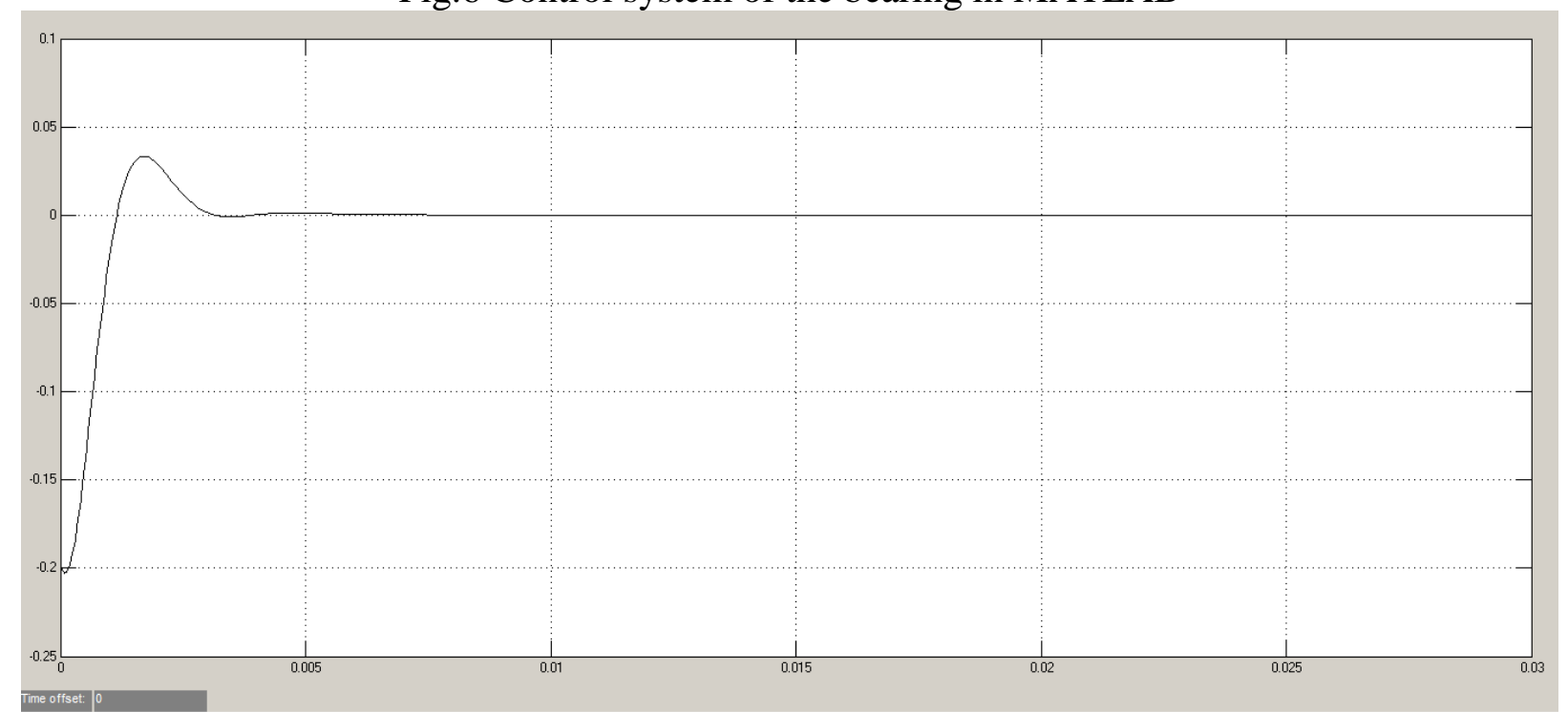

Fig.7 Result of PID controller in co-simulation

It can be seen in Fig.7 that the system can be stable with the appropriate parameters for the mathematical model, with quite different result. On the other hand also shows that the mathematical model is too ideal. The co-simulation makes the mechanical properties of the system can not be ignored, which is more instructive in practical application.

To make the system stable and have a better characteristic, a variable parameters link is added into the control system, which increases the damping of system [6]. The new system chooses different parameters according to both the error and the rate of error. The new control system is shown in Fig.8, and the comparison of the two systems with different parameters in Fig.9.

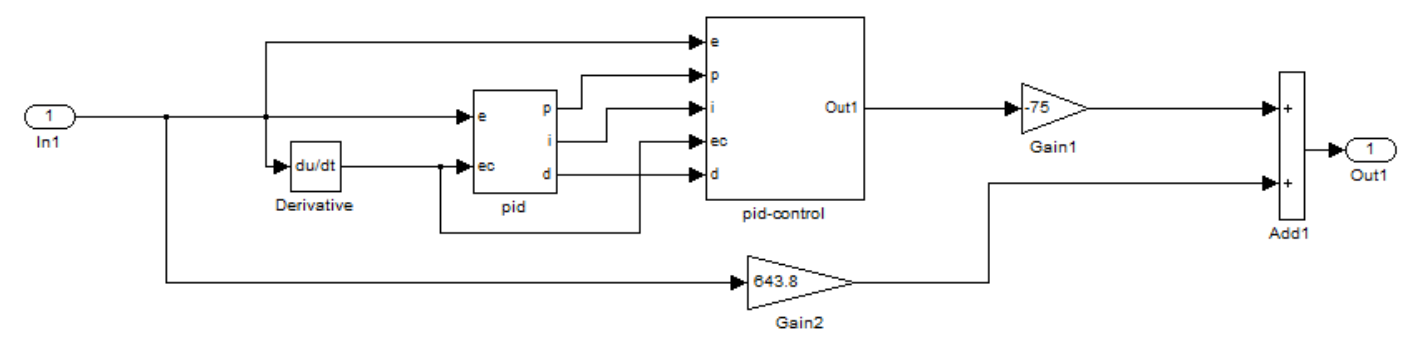

Fig.8 PID controller with variable parameters part in MATLAB 


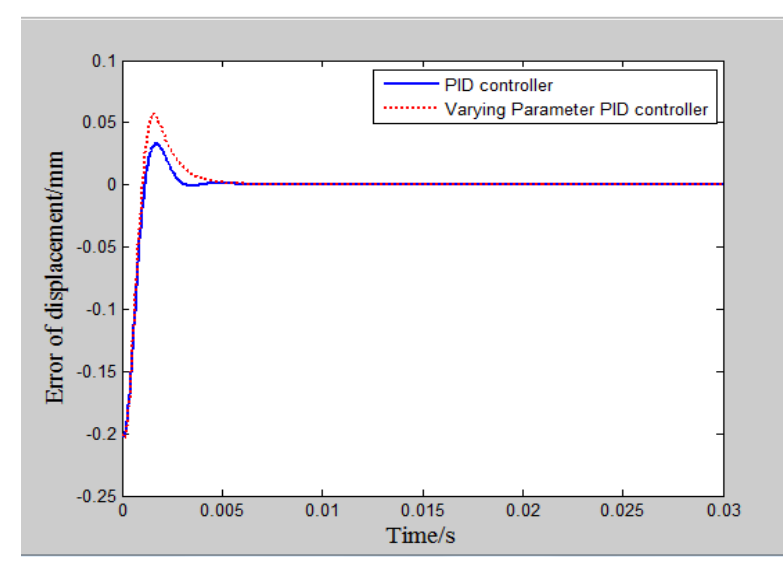

(a)with $K_{p}=-200, K_{i}=-1, K_{d}=-0.1$.

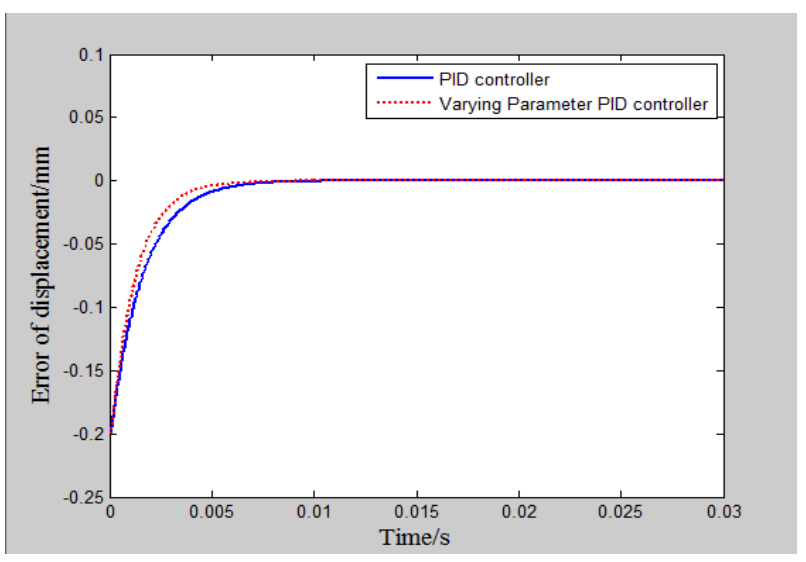

(b)with $K_{p}=-600, K_{i}=-100, K_{d}=-1$.

Fig.9 Results of PID controller and PID with variable parameters part in co-simulation

As can be seen, this PID controller with variable parameters part can make magnetic bearing keep stable and work better with suitable settings. When the system is underdamped, the variable link may choose bigger $K_{p}$ to make the system stabilize quickly, with bigger overshoot. However, the variable link makes it better in overdamped system. Because of the mechanical properties considered in the co-simulation, this control scheme can also be used in practical application.

\section{Summary}

In this paper, an idea for the study of the control system of magnetic bearings is shown, including the preparation of data and the mechanical model and the control model in the co-simulation. The PID controller with variable parameters part is simulated in MATLAB and ADAMS. The conclusion can provide a reference for the design of active magnetic bearing radial control system. The reason why results in MATLAB can not be used directly in the practical model is the mathematical model is too ideal, also too simple. Many factors are ignored in progress of solving mathematical model. More accurate models may have better results.

Further investigations will be done to study more stable control systems. PID controller is the most widely used controller. For better characteristics in actual use, other links are added into the system. Except the variable part used herein, there are many intelligent controller, such as optimum control, adaptive control, robust control and so on [7]. It is also one of the research focus of magnetic bearing.

\section{References}

[1] Huimin Li, Xixuan Wang, Current situation and development trend of magnetic bearings,Bearings, 2003(6):50-52.

[2] Heng Feng, Simulation of magnetic levitation bearing system, Nanjing University of Aeronautics and Astronautics, 2010.

[3] Hijikata K, Kobayashi S, Takemoto M, Tanaka Y, Chiba A, Fukao T. Basic Characteristics of an Active Thrust Magnetic Bearing With a Cylindrical Rotor Core[J]. IEEE TRANSACTIONS ON MAGNETICS, 2008, 44(11): 4167-4170

[4] Huayi Zhang, Analysis and Optimization of Active Magnetic Bearing, Shanghai Jiao Tong University, 2010.

[5] Pengfei Xiao, magnetic bearing system with sensor redundancy, Nanjing University of Aeronautics and Astronautics, 2012.

[6] Zhenxing Qiu, PID Controller for Active Magnetic Bearings, Beijing Jiaotong University, 2010.

[7] Junchen Song, Huimin Ouyang, Guangming Zhang, Magnetic Bearing Dynamic Analysis and Active Control, Electrical Engineering Technology, 2014,(12):165-168. 